\title{
Quality of Life in Depressed Patients in UK Primary Care: The FINDER Study
}

\author{
Alan Lenox-Smith • Mark T. B. Macdonald - Catherine Reed • \\ Andre Tylee $\cdot$ Robert Peveler $\cdot$ Deborah Quail $\cdot$ Hiram J. Wildgust
}

To view enhanced content go to www.neurologytherapy-open.com

Received: December 6, 2012 / Published online: March 13, 2013

(c) The Author(s) 2013. This article is published with open access at Springerlink.com

\section{ABSTRACT}

Objectives: To investigate the impact of depression and its treatment on health-related quality of life (HRQoL) in a naturalistic, primary care setting in the UK.

A. Lenox-Smith $(\varangle) \cdot$ M. T. B. Macdonald Eli Lilly \& Company Ltd., Lilly House, Priestley Road, Basingstoke, Hampshire RG24 9NL, UK e-mail: lenox-smithal@lilly.com

C. Reed · D. Quail

Eli Lilly \& Company Ltd., Erl Wood Manor, Windlesham, Surrey GU20 6PH, UK

\section{A. Tylee}

Health Services and Population Research Department, Section of Primary Care Mental Health, Institute of Psychiatry, De Crespigny Park, Denmark Hill, London SE5 8AF, UK

R. Peveler

Clinical Neurosciences Division, Southampton General Hospital, University of Southampton, Southampton SO16 6YD, UK

H. J. Wildgust

Hiram Consulting Ltd., 11 Cricketers Close, Ackworth, Pontefract, West Yorkshire WF7 7PW, UK

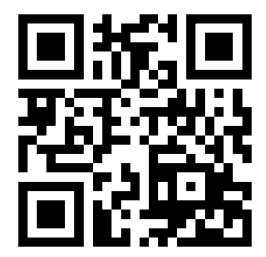

Enhanced content for this article is available on the journal web site: www.neurologytherapy-open.com
Methods: The Factors Influencing Depression Endpoints Research (FINDER) study was a European, 6-month, prospective, observational study designed to estimate HRQoL in patients with a clinical diagnosis of depression. This paper examines primary care patients recruited in the UK. HRQoL was measured at baseline and at 3 and 6 months after starting antidepressant therapy using the Short Form 36 Health Status Survey and the European Quality of Life-5 Dimensions (EQ-5D). Regression analysis was used to identify baseline and treatment variables independently and significantly associated with HRQoL. Further analyses included the effect of caseness for depression on HRQoL, the effect of moderate/severe pain at baseline on HRQoL, changes in overall pain, pain interference scores, and the use of different antidepressants by pain cohort.

Results: A total of 608 patients was recruited from 58 centres and mean HRQoL was significantly below reported population norms at baseline. Most improvement in HRQoL was seen at 3 months for EQ-5D, with small additional improvement at 6 months. Worse HRQoL outcomes at 6 months were associated with higher somatic symptoms score, duration 
of depression at baseline, and switching within antidepressant classes. Patients meeting the criteria for caseness for depression, or with significant pain at baseline showed less improvement in HRQoL scores at 6 months.

Conclusion: Patients presenting with depression in primary care show reduced HRQoL compared to population norms. HRQoL improves during antidepressant treatment particularly within the first 3 months. Nonpainful somatic symptoms, socioeconomic factors, depression variables and switching within antidepressant class predict poor HRQoL outcome. Pain is a common symptom in depressed patients and remains after 6 months' treatment. Pain and somatic symptoms should be assessed in all patients with depression in primary care.

Keywords: Antidepressant; Depression; Pain; Primary care; Quality of life

\section{INTRODUCTION}

The concept of health was defined by the World Health Organization in 1948 as "a state of complete physical, mental and social wellbeing and not merely the absence of disease or infirmity" [1]. This was later refined into five dimensions of health-related quality of life (HRQoL) encompassing physical, social, psychological, overall life satisfaction/ wellbeing and perceptions of health status at the 1993 International Consensus Conference [2]. HRQoL is used to assess both functional outcome and quality of life (QoL) across disease states and has been demonstrated to be impaired in depressed patients compared to both the general population and to people with chronic medical conditions such as diabetes, arthritis, and heart disease [3-5]. It is therefore important to understand what factors influence HRQoL in depression and how these influence response to treatment. The five dimensions of HRQoL can be assessed using tools such as the Short Form 36 Health Status Survey (SF-36) [6] and European Quality of Life-5 Dimensions (EQ-5D) [7] and although studies have previously assessed the effectiveness of antidepressant treatments on HRQoL [8-13] further research is needed in order to improve our understanding of factors related to HRQoL and its improvement during treatment. Two possible moderators of HRQoL in depression are somatic and pain symptoms. Many depressed patients present with somatic symptoms [14] and there is a strong association between depression and painful symptoms in primary care [15]. It is known that painful symptoms adversely affect treatment outcomes in depression [16], but less is known about their impact on HRQoL outcomes.

The European Factors Influencing Depression Endpoints Research (FINDER) observational study [17-19] was designed to increase understanding of the factors that influence HRQoL outcomes for clinically depressed outpatients receiving antidepressant medication in routine primary and secondary care. In this study, pain and its impact on functioning were also assessed using patientreported measures. A strength of this study is that it included patients with other chronic medical comorbidities that may influence HRQoL and outcomes, and so is more reflective of the clinical populations found in primary care than those from randomized controlled trials in which greater use of exclusion criteria is normal. The findings from naturalistic observational studies such as FINDER may therefore be generalizable to a larger population and can be used to provide direction for further research [20]. 
The primary objective of this paper is to estimate the HRQoL of a primary care depressed population at baseline (untreated) and 3 and 6 months after initiation of antidepressant treatment. A shared secondary objective was to determine the factors associated with HRQoL at baseline and 6 months. Additional, post hoc secondary objectives specific to the UK sample were: to describe the impact of caseness for depression at baseline on HRQoL; to describe the impact of pain (explained medical cause/ unexplained medical cause) at baseline on HRQoL; to describe changes in overall pain and pain interference outcomes by pain cohort (explained medical cause/unexplained medical cause); and to summarize antidepressant use by pain cohort (explained medical cause/ unexplained medical cause).

The authors are not aware of any other paper which has sought to bring together outcomes as measured by QoL with the factors that influence outcomes as described above, making this an innovative paper with a particular relevance to primary care.

\section{METHODS}

\section{Study Design}

The UK subset of the FINDER study was designed as a prospective, noninterventional, observational study to measure QoL in patients diagnosed as having depressive disorder by their general practitioner (GP) and initiated on antidepressants. The results of the full European study have already been published elsewhere [17-19]. Here, the authors report the study design and findings from the UK cohort of patients only. The study was approved by the Eastern Multicentre Research Ethics Committee in Cambridge. Subsequent to clinical diagnosis and the decision to treat with an antidepressant, patients gave written informed consent for the provision and collection of data during the observation period.

\section{Patient Sample and Data Collection}

Patients based in primary care were enrolled by participating investigators between May 2004 and September 2005 if they: presented within the normal course of care for depression and were clinically diagnosed with depression by their GP; were about to receive pharmacological treatment for either their first episode of depression or for a new episode of depression; were at least 18 years of age; and were not simultaneously participating in a different study that included an investigational drug or procedure. The choice of pharmacological treatment was determined solely at the discretion of each participating physician in agreement with the patient.

Data collection for the study took place during routine visits to the patient's GP. There were three data collection time points: at baseline, when the patient was prescribed pharmacological treatment for a new or first episode of depression; at $3( \pm 1)$ months post baseline; and at $6( \pm 1)$ months post baseline.

Baseline data collected included patient sociodemographics, psychiatric history, duration of the current depressive episode, and the presence of comorbid chronic medical conditions and functional syndromes. Antidepressants prescribed were recorded and subsequently grouped as follows: selective serotonin reuptake inhibitors (SSRIs), serotonin noradrenaline reuptake inhibitors (SNRIs), tricyclic antidepressants (TCAs), others (including herbal remedies, lithium, monoamine oxidase inhibitors), or combinations of antidepressants. 


\section{Measures}

HRQoL was completed by the patient using the two components of the SF-36 [SF-36 version 2.0: mental components summary (MCS), which includes vitality, social functioning, roleemotional and mental health scales, and physical components summary (PCS), which includes physical functioning, role-physical, bodily pain, and general health scales] [6] and the EQ-5D [7].

The Hospital Anxiety and Depression Scale [HADS; subscales HADS-A (anxiety) and HADSD (depression)] [21] was used by patients to selfrate symptoms of anxiety and depression. As patients were diagnosed with depression based on the clinical judgment of the investigator rather than with a recognized diagnostic tool, the HADS was used to define a case of probable depression. Caseness for depression was defined as a score of 8 or greater on HADS-D, which has high sensitivity and specificity for detecting a major depressive episode $(0.80$ and 0.88 , respectively) in a GP setting [22].

Somatic symptoms were assessed using the 28-item Somatic Symptom Inventory (SSI-28) [23], consisting of seven pain-related items (SSIpain) and 21 items not relating to pain (SSIsomatic).

Overall pain severity and the impact of pain on daily functioning during the past week were rated by patients at each visit using a visual analog score (VAS; 0-100). For overall pain severity, ratings of $30 \mathrm{~mm}$ or less were categorized as no/mild pain and over $30 \mathrm{~mm}$ were considered to be moderate/severe pain [24].

\section{Sample Size}

A target sample size of 600 patients was determined for the original, UK-only study based on the primary objective of determining the QoL of a depressed population before treatment and at 3 and 6 months after treatment had started using the two summaries of SF-36: the MCS and the PCS. According to a SF-36 user manual [25], the mean (SD) MCS and PCS for a clinically depressed population (in the US) are 34.84 (12.17) and 44.96 (12.05), respectively. For the normal population, the mean (SD) is 50 (10) for both MCS and PCS. Assuming a SD of approximately 12 with 600 patients, the $95 \%$ confidence limits around the mean were calculated to be \pm 0.96 for both the MCS and PCS.

\section{Statistical Analysis}

Descriptive statistics were calculated for each variable for all patients eligible for analysis at baseline; only those patients with data from at least one follow-up visit were included in the regression analyses.

\section{HRQoL and Independent Variables}

The baseline analysis of HRQoL used linear regression analysis to identify independent variables associated with HRQoL; a mixedeffects model repeated measures analysis with unstructured covariance structure was used for the longitudinal analyses. Backward regression methods were used to identify variables independently associated with HRQoL outcomes. Separate models were fitted for each of the following outcome variables: SF-36 (MCS, PCS), EQ-5D [VAS and health status index (HSI)]. Independent variables were removed from the full model until only statistically significant $(P \leq 0.05)$ variables remained. The independent variables included in the initial model were: age, gender, education (none/mandatory, further), occupation status (working for pay, unemployed, other), marital status (married/ domestic partner, other), body mass index, 
number of dependants, smoking (yes, no), number of previous episodes of depression, age at first episode, any psychiatric illness in the 24 months before baseline (yes, no), any functional syndromes (yes, no), duration of current depressive episode, HADS-A score, HADS-D score, SSI-somatic score, and pain cohort (no/mild pain, explained pain, unexplained pain). Also included in the longitudinal analysis were: class of antidepressant taken between 3 and 6 months, switch (between, within, none) between antidepressants taken between baseline and 3 months and between 3 and 6 months, and baseline value of the dependent variable.

\section{HRQoL and the Effect of "Caseness" of Depression}

HRQoL scores are divided into two depression subgroups: cases of depression and noncases of depression, as defined at baseline by the HADSD score. A score of 8 or greater indicates a case of depression, whereas a score of less than 8 indicates a noncase.

\section{HRQoL and the Effect of Pain}

Three pain subgroups are described: those with no or mild pain; those with moderate/severe pain with a defined medical disorder known to cause pain as based on physician selection of listed comorbidities (termed explained pain); and those with moderate/severe pain with a defined medical disorder not associated with pain or without further comorbidity other than depression (termed unexplained pain). Pain was assessed in the study using the overall pain VAS, in which a score of $0-30$ represented no/mild pain and a score over 30 represented moderate/ severe pain. For HRQoL and VAS pain scores in the overall sample and in the three pain cohorts, if the 95\% two-sided confidence intervals (CI) for the means at two time points or for two cohorts did not overlap, the difference was considered statistically significant as this procedure corresponds to a conservative two-sided test at the 5\% level.

\section{RESULTS}

A total of 608 patients was enrolled in the study in the UK by 58 participating doctors, of whom 57 were GPs.

\section{Patient Characteristics at Baseline}

The baseline characteristics of eligible patients are summarized in Table 1. A total of $35 \%$ had a history of depressive episodes in the previous 2 years (excluding the index episode), and anxiety/panic disorder (29\%) was the most common psychiatric illness experienced in the 24 months before baseline. At baseline, 34\% of patients had at least one comorbid, chronic, physical condition and 19\% had a comorbid functional syndrome (19\%). The most common comorbid physical conditions were hypertension (13\%) and asthma (11\%), and the most common comorbid functional syndrome was irritable bowel syndrome (10\%). At baseline, $82 \%$ of patients met the criteria for a case of depression (HADS-D score $\geq 8$ ). At the baseline visit, $68 \%$ of patients had not received an antidepressant in the previous 24 months, $21 \%$ had received SSRIs only, $1 \%$ TCAs only, $5 \%$ other drugs only, and 5\% combinations of antidepressants.

\section{Health-Related Quality of Life}

Figure $1[25,26]$ shows the mean [standard deviation (SD)] SF-36 scores (MCS and PCS) and mean (SD) EQ-5D scores (VAS and HSI) at baseline, 3 and 6 months. At baseline SF-36 (MCS), EQ-5D VAS and EQ-5D HSI scores were 
Table 1 Baseline characteristics of patients $(n=608)$

\begin{tabular}{ll}
\hline Variable & \% or mean \pm SD \\
\hline Age (years) & $42.8 \pm 14.7$ \\
Women & 61.2 \\
Body mass index $\left(\mathrm{kg} / \mathrm{m}^{2}\right)$ & $26.9 \pm 6.2$ \\
Duration of depression (years) & $5.3 \pm 8.3$ \\
History of depressive episodes & 34.6 \\
in previous 24 months & \\
(excluding index episode) $^{2}$ & \\
Duration of current major & $15.7 \pm 17.9$ \\
depressive disorder & \\
episode (weeks) & \\
Other psychiatric illnesses & \\
in previous 24 months & \\
Anxiety/panic disorder & \\
Obsessive compulsive disorder & 4.5 \\
Drug/alcohol dependence & 6.0 \\
$\quad$ disorder & \\
Pain severity cohort & \\
No/mild pain & \\
Explained pain & \\
Unexplained pain & \\
\hline
\end{tabular}

$S D$ standard deviation, $V A S$ visual analog scale

${ }^{a}$ Reported if greater than 4\%. Examples below this threshold include schizophrenia and bipolar disorder

b Pain severity was rated by patients on a $100 \mathrm{~mm}$ VAS in which $0=$ no pain and $100=$ pain as severe as I can imagine

${ }^{c}$ Overall pain VAS $\leq 30$

d Overall pain VAS $>30$

significantly below general population norms. There were improvements in HRQoL (except SF-36 PCS) at 3 months with further small improvements at 6 months.

The independent variables significantly associated with HRQoL at baseline are summarized in Table 2 and include SSI-somatic score, HADS-D, HADS-A, presence of moderate to severe pain, gender, occupational status, and age.
The independent variables significantly associated with changes in HRQoL from baseline to 6 months are shown in Table 3 . Numerous factors were associated with a worse HRQoL outcome: a higher SSI-somatic score at baseline, socioeconomic factors (older age, being unemployed, being married), depression variables (higher HADS-D score at baseline, more previous episodes of depression, longer duration of current depressive episode), previous psychiatric illness, comorbid functional syndromes, and treatment switching within antidepressant class during the 6-month treatment period. Factors associated with a better HRQoL outcome include female gender, older age at first episode, and higher HRQoL scores at baseline.

\section{HRQoL by Caseness for Depression}

Figure $2[25,26]$ presents the mean HRQoL results (SF-36 MCS and PCS, EQ-5D VAS and HSI) by caseness for depression (baseline HADS$\mathrm{D}$ score $\geq 8$ indicates a case of depression). Cases of depression were associated with lower mean HRQoL scores at all time points, compared with noncases. There were increases in the mean SF-36 MCS and EQ-5D scores during treatment in both cases and noncases of depression. SF-36 PCS scores were within one SD (10) of the population norm at baseline and during the course of treatment for both cases and noncases.

\section{HRQoL by Pain Cohort}

Figure $3[25,26]$ presents the mean HRQoL results by pain cohort. Patients with pain (explained pain and unexplained pain) had lower mean scores for SF-36 PCS and EQ-5D (HSI and VAS) at all three time points than patients with no/mild pain. Notably, the mean PCS scores remained at or below clinical depression norms even after 6 months of treatment in the two groups with pain. 


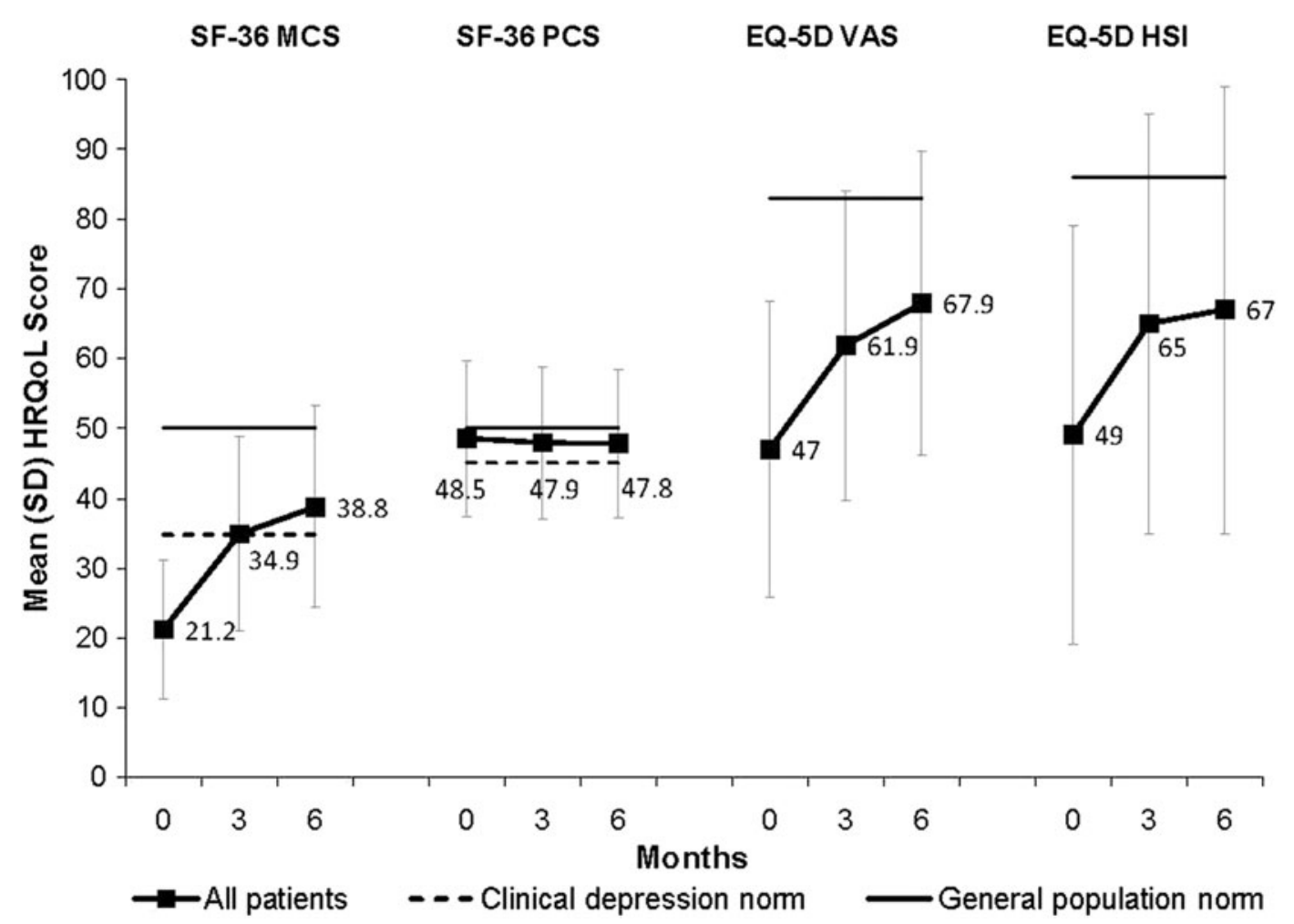

Fig. 1 Mean (SD) SF-36 and EQ-5D scores at baseline, 3 and 6 months. SF-36 MCS and PCS scores were normalized to a mean of 50 (SD 10). The solid line represents the SF-36 general US population norm (mean 50) and the dotted line represents the clinical depression norms (MCS mean 34.8; PCS mean 45.0) [25]. Mean EQ-5D HSI scores were converted from a $0-1$ scale to a $0-100$ scale. The solid

\section{Pain Outcomes}

The mean pain VAS for all patients at baseline was 34.1 (95\% CI 31.8-36.3). This decreased to 29.9 (95\% CI 27.3-32.5) at 3 months and to 27.1 (95\% CI 24.4-29.9) at 6 months; the decrease from baseline to 6 months was considered significant as the $95 \%$ CIs did not overlap. The decrease from baseline was not significant at 3 months.

Table 4 shows the VAS scores for overall pain and interference of pain with ability to perform daily activities by pain cohort at baseline, 3 and 6 months.

The mean overall pain VAS (i.e., pain severity) was significantly reduced from line represents the UK general population norms (EQ-5D VAS mean 82.5; EQ-5D HSI mean 86) [26]. EQ-5D European Quality of Life-5 Dimensions, HSI health status index, $H R Q O L$ health-related quality of life, MCS mental component summary, PCS physical component summary, $S D$ standard deviation, $S F-36$ Short Form 36 Health Status Survey, $V A S$ visual analog scale

baseline at 3 and 6 months in both the explained and unexplained pain cohorts. Mean VAS scores for interference of pain with ability to do daily activities were reduced from baseline at 3 and 6 months for both the explained and unexplained pain cohorts, indicating that pain interfered less with patients' ability to do daily activities. This change was significant for the unexplained pain group only.

\section{Treatment Patterns by Pain Cohort}

Figure 4 presents the antidepressant use patterns for the first and second 3 months of treatment in the three pain cohorts (no/mild 


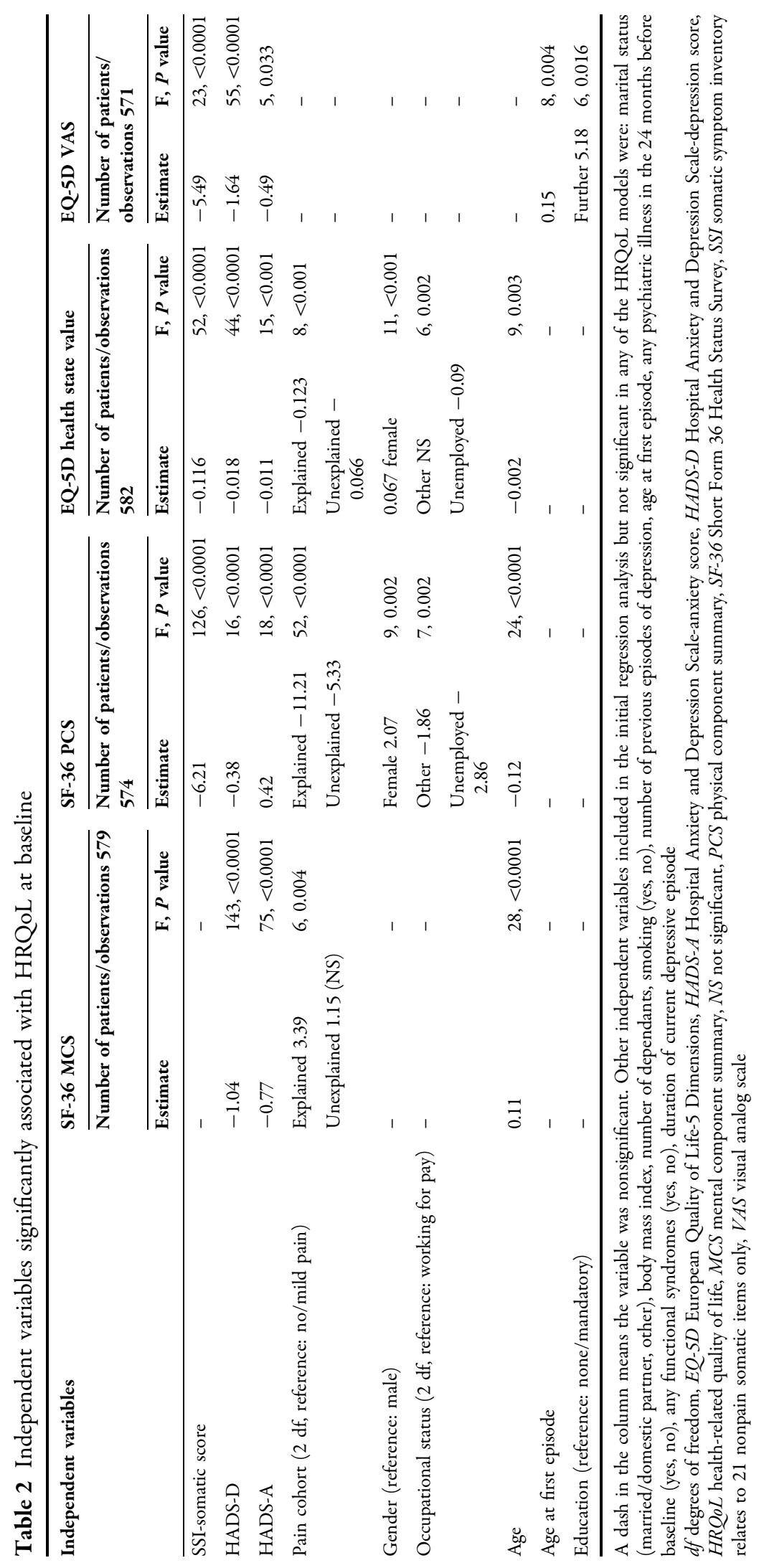




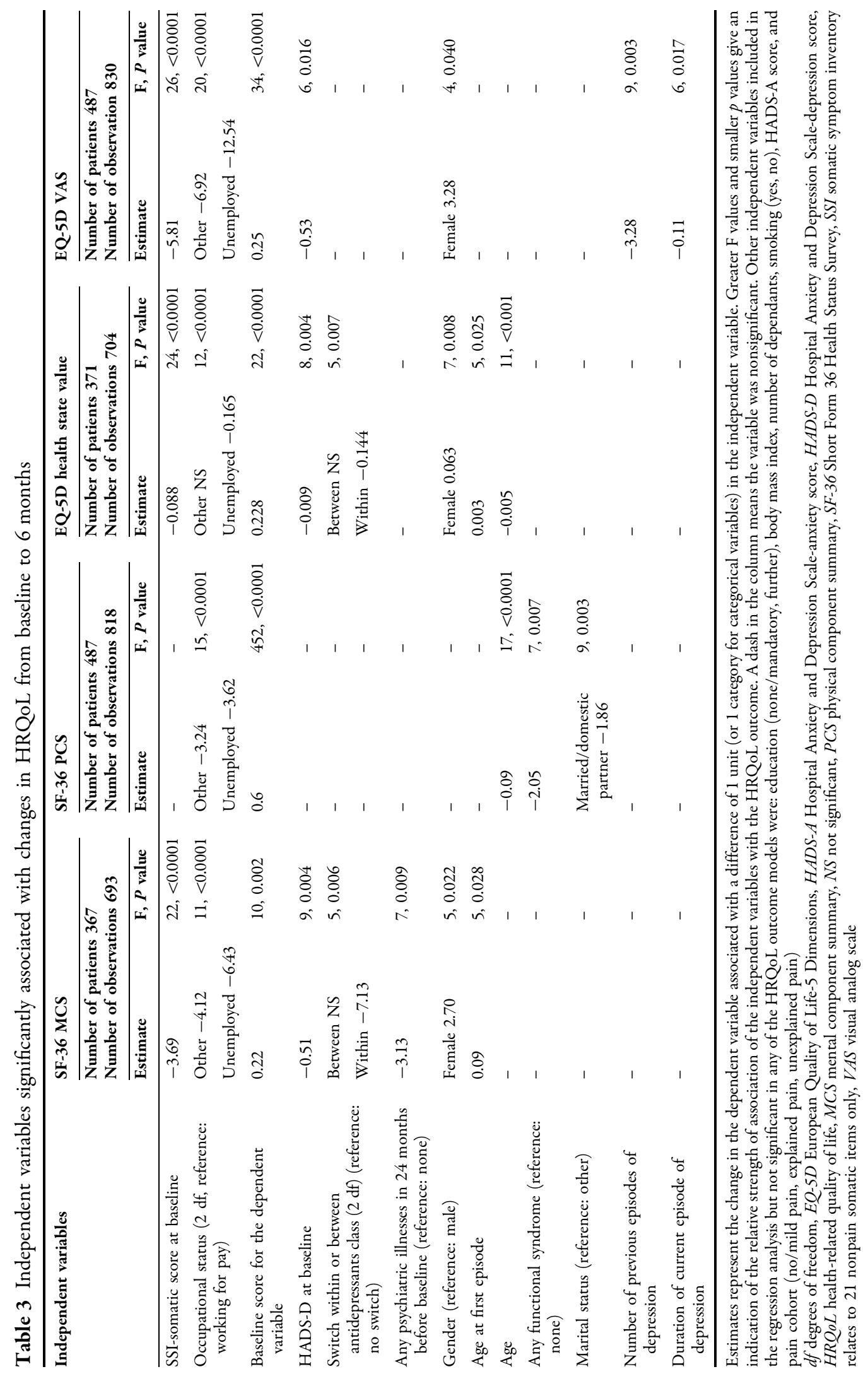




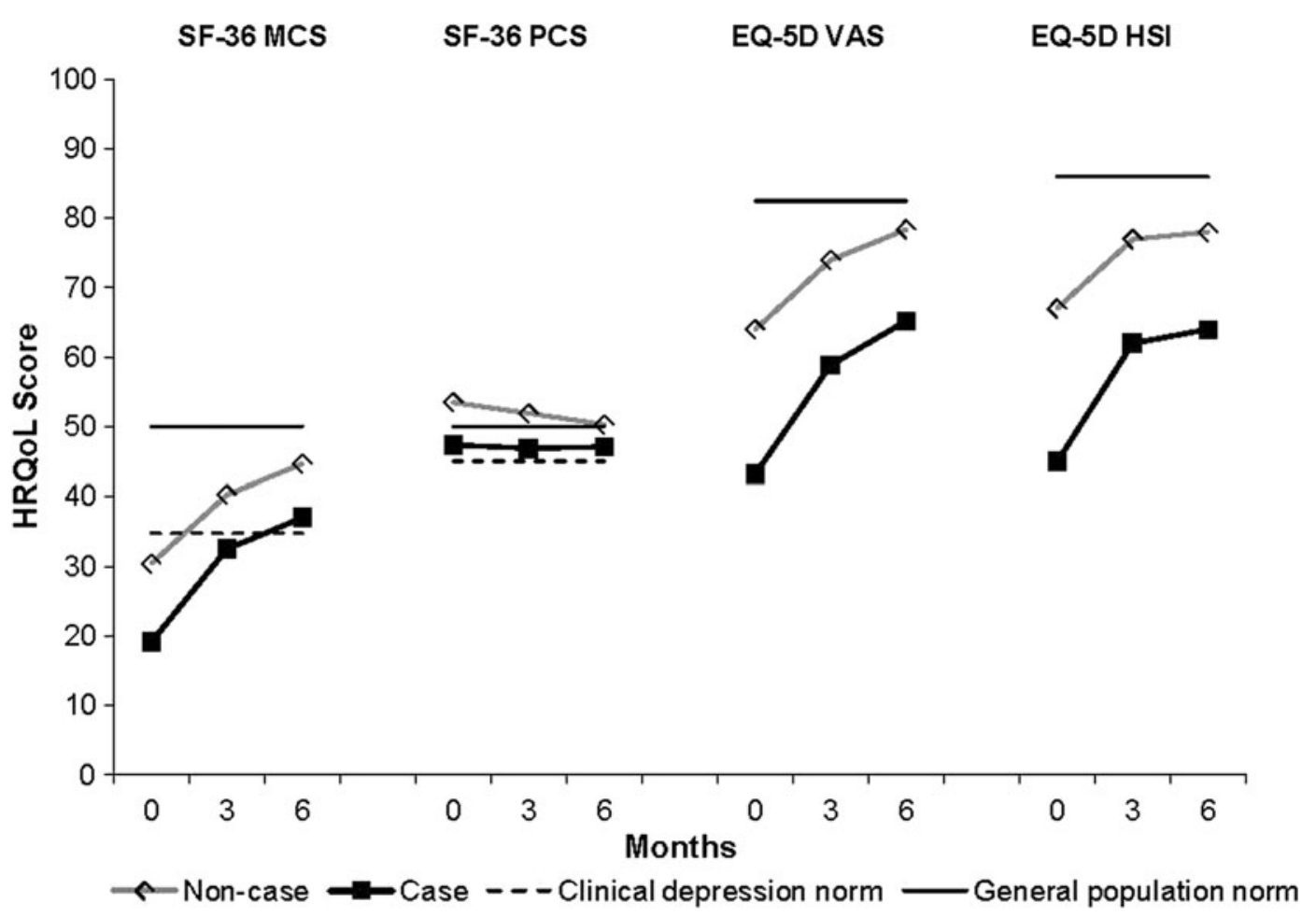

Fig. 2 Mean HRQoL scores at baseline, 3 and 6 months by caseness for depression (based on HADS-D score at baseline: score $\geq 8=$ case; score $<8=$ noncase). SF-36 MCS and PCS scores were normalized to a mean of 50 (SD 10). The solid line represents the SF-36 general US population norm (mean 50) and the dotted line represents the clinical depression norms (MCS mean 34.8; PCS mean 45.0) [25]. Mean EQ-5D HSI scores were converted from a $0-1$ scale to a $0-100$ scale. The solid line represents the UK

pain, explained pain, and unexplained pain) as defined at baseline. Note, this is actual use of medication during 0-3 months and 3-6 months as reported by patients, not the medication prescribed at the baseline and 3-month visits, respectively. SSRIs were the most commonly used antidepressants irrespective of pain symptoms, although during the second 3 months of observation there was a decrease in their use that was highest in the explained pain cohort.

Prescribing patterns were very similar for the cohorts with no/mild pain and unexplained pain suggesting little differential prescribing general population norms (EQ-5D VAS mean 82.5; EQ-5D HSI mean 86) [26]. EQ-5D European Quality of Life-5 Dimensions, HADS-D Hospital Anxiety and Depression Scale-depression, HSI health status index, HRQoL healthrelated quality of life, $M C S$ mental component summary, $P C S$ physical component summary, $S D$ standard deviation, SF-36 Short Form 36 Health Status Survey, VAS visual analog scale

based on the presence of painful symptoms. It is also interesting to note that during the second 3 months of treatment, $15-22 \%$ of patients reported not using any antidepressants.

\section{DISCUSSION}

The results of this study found that HRQoL (as measured by the SF-36 and EQ-5D) in patients with a first or new episode of depression presenting in UK primary care was below that previously reported in healthy populations. HRQoL improved during the first 3 months following antidepressant initiation, with more 


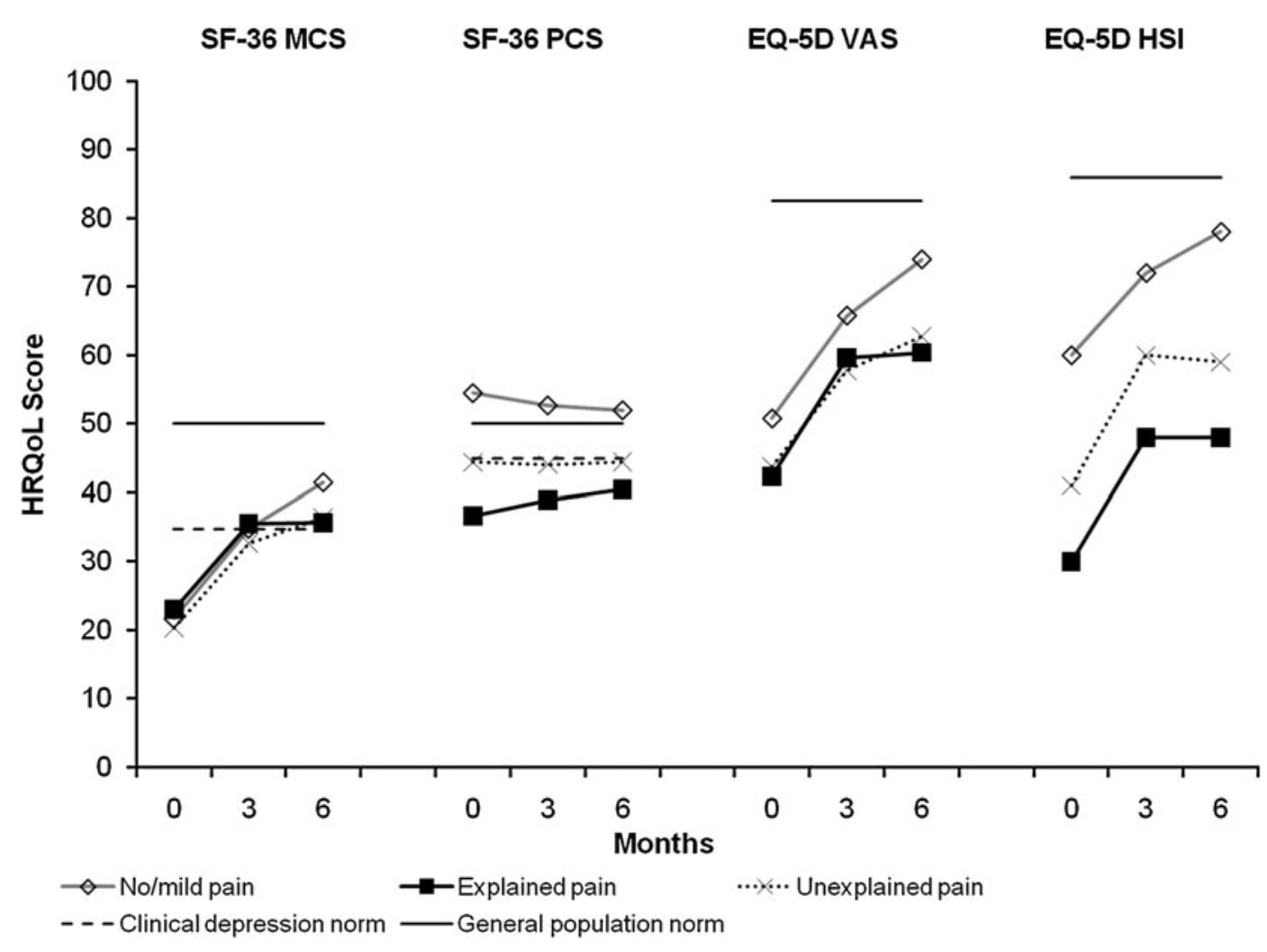

Fig. 3 Mean HRQoL scores at baseline, 3 and 6 months by pain cohort. SF-36 MCS and PCS scores were normalized to a mean of 50 (SD 10). The solid line represents the SF36 general US population norm (mean 50) and the dotted line represents the clinical depression norms (MCS mean 34.8; PCS mean 45.0) [25]. Mean EQ-5D HSI scores were converted from a $0-1$ scale to a $0-100$ scale. The solid line represents the UK general population norms (EQ-5D VAS

limited improvement from 3 to 6 months. The findings of this study in UK primary care patients are therefore similar to those of the full European FINDER study with respect to the primary objective. Similarly, the SF-36 MCS score at the end of the study had not normalized (50) and was at least 1 SD lower than for the general population. The study also showed minimal change on SF-36 PCS, probably reflecting a ceiling effect as a mean score of 50 represents the score for the general population. These results are consistent with other clinical studies [8-13].

The independent variables significantly associated with HRQoL at baseline in the UK mean 82.5; EQ-5D HSI mean 86) [26]. EQ-5D European Quality of Life-5 Dimensions, EX/P explained pain, $H S I$ health status index, HRQoL health-related quality of life, $M C S$ mental component summary, $N / M P$ no/mild pain, $P C S$ physical component summary, $S D$ standard deviation, SF-36 Short Form 36 Health Status Survey, UNEX/P unexplained pain, $V A S$ visual analog scale

sample include SSI-somatic score, HADS-D, HADS-A, pain cohort, gender, occupational status, and age. Patients with adverse sociodemographics, greater general medical and depressive illness burden have been identified with poorer QoL [27]. The authors' finding that a higher SSI-somatic score was associated with a poorer HRQoL supports previous findings of an inverse relationship between somatic symptoms and QoL [28]. Moreover, a recent cross-sectional study in a large population of primary care patients with depression showed that numerous characteristics of somatic symptoms (number, 
Table 4 VAS scores for pain at baseline, 3, and 6 months by pain cohort

\begin{tabular}{|c|c|c|c|c|c|c|}
\hline & \multicolumn{2}{|c|}{ No/mild pain } & \multicolumn{2}{|c|}{ Explained pain } & \multicolumn{2}{|c|}{ Unexplained pain } \\
\hline & $n$ & Mean $(95 \% \mathrm{CI})$ & $n$ & Mean $(95 \% \mathrm{CI})$ & $n$ & Mean $(95 \% \mathrm{CI})$ \\
\hline \multicolumn{7}{|c|}{ Overall pain ${ }^{a}$} \\
\hline Baseline & 297 & $9.5(8.4-10.6)$ & 65 & $62.3(57.9-66.6)$ & 239 & $57.0(54.8-59.2)$ \\
\hline 3 months & 216 & $16.2(13.3-19.1)$ & 46 & $46.0(38.0-53.9)$ & 173 & $42.4(38.4-46.4)$ \\
\hline 6 months & 192 & $14.8(12.2-17.4)$ & 43 & $48.0(38.6-57.3)$ & 155 & $36.4(31.6-41.2)$ \\
\hline \multicolumn{7}{|c|}{ Interference with daily activities ${ }^{\mathrm{b}}$} \\
\hline Baseline & 296 & $12.8(10.5-15.0)$ & 65 & $60.7(53.0-68.4)$ & 239 & $53.9(50.2-57.6)$ \\
\hline 3 months & 215 & $12.5(9.7-15.2)$ & 46 & $45.6(35.9-55.2)$ & 174 & $36.4(31.8-41.1)$ \\
\hline 6 months & 192 & $10.2(7.8-12.7)$ & 43 & $44.1(33.3-55.0)$ & 155 & $33.9(28.5-39.3)$ \\
\hline
\end{tabular}

No/mild pain was defined as an overall pain VAS $\leq 30$ at baseline; both the explained and unexplained pain groups had an overall pain VAS $>30$ at baseline

$C I$ confidence interval, $V A S$ visual analog scale

${ }^{a}$ Overall pain severity was rated by patients on a $100 \mathrm{~mm}$ VAS in which $0=$ no pain and $100=$ pain as severe as I can imagine

b Interference of overall pain with ability to perform daily activities was rated by patients on a $100 \mathrm{~mm}$ VAS with $0=$ not at all to $100=$ complete

disability, persistence) were associated with a decreased QoL [29].

Higher SSI-somatic scores were also associated with worse HRQoL outcomes over time, highlighting the continuing impact of (nonpainful) somatic symptoms on HRQoL. Somatic symptoms have been found to be associated with pain and improvement in pain outcomes in the European cohort of the FINDER study [30]. As the presence of painful symptoms has been shown to compromise outcomes of antidepressant treatment [16, 31], so the results of this study suggest that nonpainful physical symptoms as well as painful symptoms should be taken into account when maximizing treatment response in depression.

An important finding was that those patients who switched antidepressant treatment within the same antidepressant class (e.g., SSRI) had a poorer HRQoL (SF-36 MCS, EQ-5D health state value) outcome over the 6-month follow-up period than those who had no change in treatment or who switched to a different antidepressant class. Switching between antidepressant classes (e.g., SSRI to SNRI) was not significantly associated with poorer HRQoL outcome. These findings suggest that if a change in treatment is necessary, it may be more advantageous in terms of HRQoL to switch from one antidepressant class to another. This should be explored in more detail in a controlled setting.

The association of sociodemographic and depression-related factors with changes in HRQoL remain consistent with the European analysis [19]. Pain cohort (no pain or either explained or unexplained pain) was not significantly associated with change in HRQoL in the UK sample, despite significant results in the univariate analysis and an association with baseline HRQoL. Pain was associated with worse HRQoL (SF-36 MCS only) in the European analysis $(P=0.026)$ using the overall pain VAS score rather than pain cohorts [19]. It is likely 


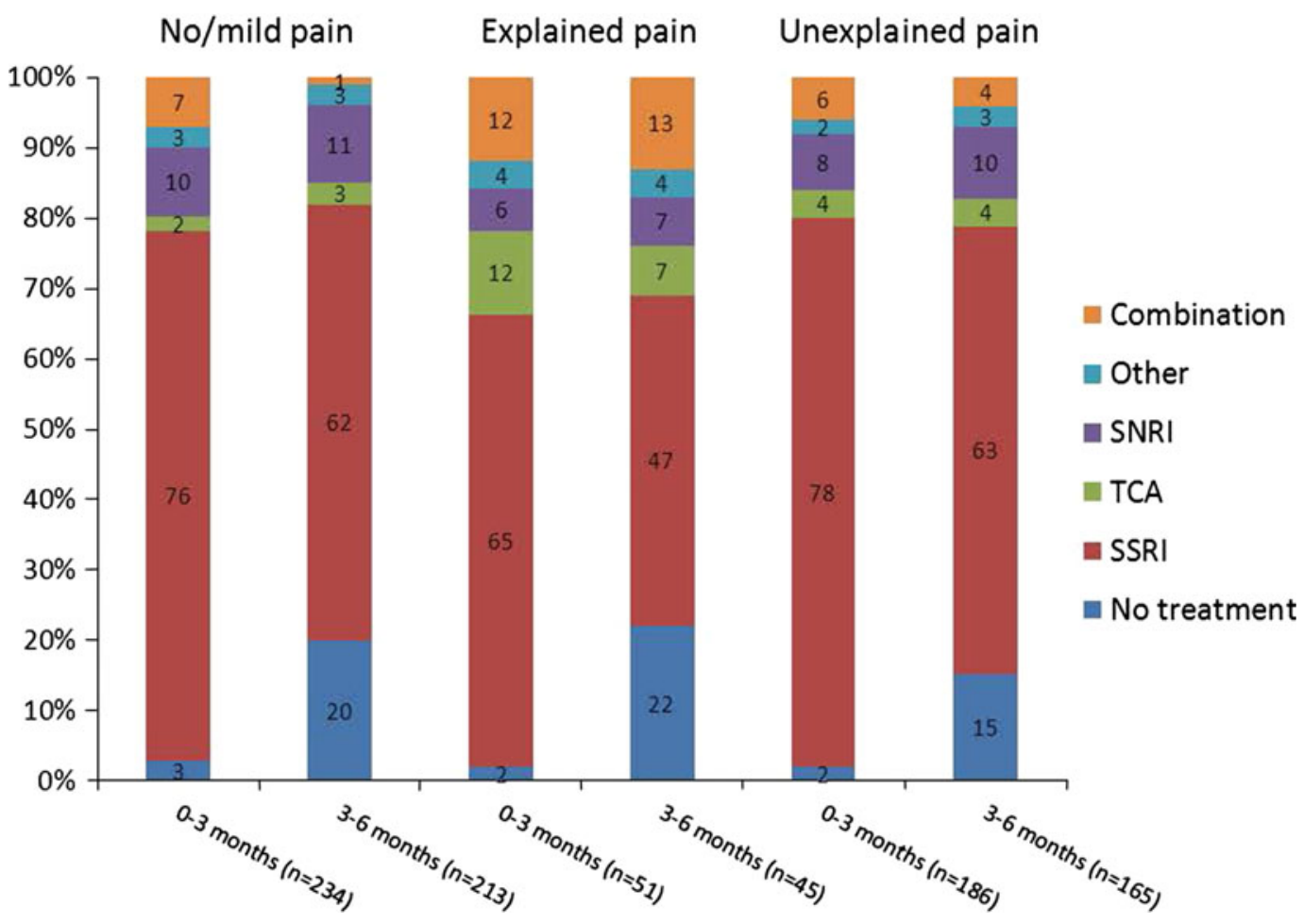

Fig. 4 Antidepressant use in the first 3 months (0-3 months) and second 3 months (3-6 months) of treatment by pain cohort. Clinically significant pain was defined as overall pain VAS greater than $30 \mathrm{~mm}$ at baseline.
SNRI serotonin noradrenaline reuptake inhibitor, SSRI selective serotonin reuptake inhibitor, TCA tricyclic antidepressant, $V A S$ visual analog scale

Using the patient-rated HADS-D score of 8 or greater as a cut-off due to high sensitivity and specificity in general practice [22], the majority of patients $(82 \%)$ recruited into the UK FINDER study met the criteria for caseness for depression. This supports the view that UK GPs involved with this study were good at diagnosing clinical depression. The baseline mean SF-36 summary scores (MCS 19.2 and PCS 47.4) for patients who met the criteria for caseness correspond closely to the mean values reported in a French primary care study [32] of patients with major depressive disorder defined by the Diagnostic and Statistical Manual of Mental Disorders, IVth revision (MCS 21.1 and PCS 43.6).

The reduction in pain severity (measured using the overall pain VAS) for the whole 
patient cohort was only statistically significant at the 6-month post baseline assessment. However, when patients with no/mild pain were separated from those with moderate/severe pain, there were significant reductions in the overall pain VAS score at both 3 and 6 months in patients with medically explained and those with unexplained pain, which exceeded the $12 \mathrm{~mm}$ threshold for a minimum clinically significant difference reported by Kelly [34]. Despite the above finding, the cohort with unexplained pain at baseline still had moderate pain at the end of the study (mean overall pain VAS 36.4). Furthermore, pain interference with daily activities was still significantly impaired in the unexplained pain cohort compared with the no/ mild pain cohort (mean VAS 33.9 vs. 10.2). This observation suggests that by 6 months, the antidepressant treatments that were given in the study (which were mainly SSRIs) were neither very effective at treating the painful somatic symptoms in depression nor at minimising the associated disability.

Antidepressant use patterns in this UK general practice study were in accordance with the National Institute for Health and Clinical Excellence guidelines for depression [35], which were published halfway through recruitment into this study (May 2004 to September 2005); the majority of patients received SSRIs as firstline treatment. The pattern of antidepressant use was similar for patients with unexplained pain and without pain, in contrast to the explained pain cohort, in which TCAs were more commonly used in the first 3 months and combination therapy in the second 3 months. This lack of differentiation indicates that GPs either do not take pain into account when selecting antidepressants for depressed patients when there is no comorbid medical condition present or feel that using different classes of antidepressant would not be of value.
This study supports the view that different antidepressant strategies may be required to reduce both disability and residual pain symptoms to normal values in the management of depressed patients with painful somatic symptoms. A recent Sequenced Treatment Alternatives to Relieve Depression (STAR-D) report [36] focusing on poorer treatment outcome with an SSRI when painful physical symptoms are present reinforces this point. Furthermore, assessment and treatment of depression in primary care should incorporate pain measurement. This could be encouraged in the UK by the adoption of pain assessment in the mental health section of the quality and outcomes framework [37] that provides voluntary targets to GPs with financial incentives.

Research into the association of painful symptoms of depression and poorer HRQoL outcome warrants further investigation in a controlled setting, in longer studies, and using different treatment modalities including comparisons of different classes of antidepressant.

The most significant strength of the study is that it is one of the largest prospective, observational studies of HRQoL during treatment for depression in a primary care setting. It reflects the reality of prescribing and outcomes in UK general practice over a 6-month time period. Furthermore, the use of robust validated measures exploring depression caseness, HRQoL and pain add to the existing literature and can help inform clinical practice and future research.

The study has several limitations in addition to those common to observational studies due to the lack of randomization, such as selection and observer bias. First, the observation period was limited to 6 months, so it is unclear if HRQoL would become comparable to population norms with further treatment, or if a deficit would remain for those with pain on 
current treatment. Conversely, due to the episodic nature of depression, it is possible that improvement in depression and related HRQoL in some subjects over the 6-month period represents the natural course of the illness rather than improvement related to any treatment given. Furthermore, without a control group, improvement could represent regression towards the mean. Second, during the second 3-month period of the study, $15-22 \%$ of patients in each group were not receiving drug treatment, and it is unclear how this may have affected HRQoL outcome overall and in the different pain cohorts. Finally, the results do not reflect the complete spectrum of patients presenting with depression in primary care, but those in whom mutual agreement between clinician and patient has resulted in a course of antidepressants. The ThREshold for AntiDepressant response (THREAD) study [38] conducted in UK general practice found that many patients, albeit with mild to moderate depression, declined to receive antidepressants.

The UK GP patients in this study, in contrast with the mixed primary and secondary care populations of the European FINDER study [17], presented with a shorter history of depression: mean duration of depression (5.3 vs. 8.5 years), mean number of depressive episodes (1.3 vs. 1.8) in the previous 24-month period for those with at least one previous episode, and a less frequent history of anxiety/panic disorder $(28.7 \%$ vs. $51.1 \%$ ) in the previous 24 -month period.

\section{CONCLUSION}

Patients who present with depression in primary care in the UK have a poor QoL, which improves markedly in the first 3 months of treatment. There are several variables associated with a poor HRQoL outcome, notably somatic symptoms (nonpainful), socioeconomic and depression factors, and switching within antidepressant class. The presence of pain with either a known or unknown cause is common, remains clinically significant, and is associated with poor QoL outcome at baseline, but is not predictive of it at 6 months. Nonpainful somatic symptoms are associated with HRQoL and pain outcomes over the treatment period. Therefore, although the relationship is complex, pain and nonpainful somatic symptoms should be assessed and monitored in all patients with depression in primary care.

\section{ACKNOWLEDGMENTS}

The FINDER study was funded by Eli Lilly and Co. Ltd., Windlesham, UK and Boehringer Ingelheim GmbH, Ingelheim, Germany. Eli Lilly and Co. Ltd. was involved in all stages of the study design, data collection, data analysis, manuscript preparation and publication decisions. Dr. Lenox-Smith is the guarantor for this article, and takes responsibility for the integrity of the work as a whole. The authors take full responsibility for the content of the paper but thank Roben Das Gupta from Boehringer Ingelheim Ltd. for his early input into the manuscript, Deirdre Elmhirst, PhD, for her medical writing services in preparing the first draft of the manuscript and Andy Bradley (Lilly) for editing assistance.

Conflict of interest. Dr. Alan Lenox-Smith, Mark Macdonald, Catherine Reed and Deborah Quail are all employees of Lilly UK. Hiram Wildgust is an ex-employee and receives consultancy fees from Lilly UK. Professor Andre Tylee is partly funded by the National Institute for Health Research Biomedical 
Research Centre for Mental Health at the South London and Maudsley NHS Foundation Trust and Institute of Psychiatry, King's College London, and has received research funding and fees for speaking and attending advisory boards over the past 20 years from Eli Lilly, Boehringer Ingelheim, Servier, Wyeth, GSK, Duphar, Boots, Pfizer, Organon, Squibb and Lundbeck. Professor Robert Peveler has received fees for speaking and/or consultancy from Lilly, Wyeth, Bristol Myers Squibb, Astra Zeneca and Servier. No other conflict of interest is reported.

Author contribution. Alan Lenox-Smith was involved in the interpretation of data and edited the manuscript and coordinated the critical review of the manuscript. Mark Macdonald undertook interpretation of the data and drafted the manuscript. Catherine Reed contributed to the concept and design, analysis and interpretation and critical review of the manuscript. Andre Tylee and Robert Peveler contributed to the concept and design of the study, interpretation of the data and critical review of the manuscript. Deborah Quail participated in the study design and performed the statistical analysis and critical review of the manuscript. Hiram Wildgust supported interpretation of the data and helped to draft the manuscript. All authors have read and approved the final manuscript.

Open Access. This article is distributed under the terms of the Creative Commons Attribution Noncommercial License which permits any noncommercial use, distribution, and reproduction in any medium, provided the original author(s) and the source are credited.

\section{REFERENCES}

1. WHO. Preamble to the Constitution of the World Health Organization as adopted by the
International Health Conference, New York, 19-22 June, 1946; signed on 22 July 1946 by the representatives of 61 states (Official Records of the World Health Organization, no. 2, p. 100) and entered into force on 7 April 1948. http://www. who.int/governance/eb/who_constitution_en.pdf. Accessed 14 Feb 2013.

2. Patrick DL, Erickson P. Health status and health policy: quality of life in health care evaluation and resource allocation. New York: Oxford University Press; 1993.

3. Hays RD, Wells KB, Sherbourne CD, Rogers W, Spritzer K. Functioning and well-being outcomes of patients with depression compared with chronic general medical illnesses. Arch Gen Psychiatry. 1995;52:11-9.

4. Wells KB, Sherbourne CD. Functioning and utility for current health of patients with depression or chronic medical conditions in managed, primary care practices. Arch Gen Psychiatry. 1999;56: 897-904.

5. Wells KB, Stewart A, Hays RD, et al. The functioning and well-being of depressed patients. Results from the Medical Outcomes Study. JAMA. 1989;262: 914-9.

6. Ware JE, Kosinski M, Dewey JE. How to score version 2 of the SF-36 ${ }^{\circledR}$ health survey. Lincoln: QualityMetric Incorporated; 2000.

7. Brooks R, EuroQol Group. EuroQol: the current state of play. Health Policy. 1996;37:53-72.

8. Kroenke K, West SL, Swindle R, et al. Similar effectiveness of paroxetine, fluoxetine, and sertraline in primary care. A randomized trial. JAMA. 2001;286:2947-55.

9. Miller IW, Keitner GI, Schatzberg AF, et al. The treatment of chronic depression, part 3: psychosocial functioning before and after treatment with sertraline or imipramine. J Clin Psychiatry. 1998;59:608-19.

10. Detke MJ, Lu Y, Goldstein DJ, McNamara RK, Demitrack MA. Duloxetine $60 \mathrm{mg}$ once daily dosing versus placebo in the acute treatment of major depression. J Psychiatr Res. 2002;36:383-90.

11. Dunner DL, Kwong WJ, Houser TL, Richard NE, Donahue RM, Khan ZM. Improved health-related quality of life and reduced productivity loss after treatment with bupropion sustained release: a study in patients with major depression. Prim Care Companion J Clin Psychiatry. 2001;3:10-6.

12. Walker V, Streiner DL, Novosel S, Rocchi A, Levine MA, Dean DM. Health-related quality of life in patients with major depression who are treated 
with moclobemide. J Clin Psychopharmacol. 1995;15(4 Suppl. 2):60-7.

13. Peveler R, Kendrick T, Buxton $M$, et al. A randomised controlled trial to compare the costeffectiveness of tricyclic antidepressants, selective serotonin reuptake inhibitors and lofepramine. Health Technol Assess. 2005;9:1-134.

14. Simon GE, VonKorff M, Piccinelli M, Fullerton C, Ormel J. An international study of the relation between somatic symptoms and depression. N Engl J Med. 1999;341:1329-35.

15. Garcia-Cebrian A, Gandhi P, Demyttenaere $\mathrm{K}$, Peveler R. The association of depression and painful physical symptoms - a review of the European literature. Eur Psychiatry. 2006;21: 379-88.

16. Bair MJ, Robinson RL, Eckert GJ, Stang PE, Croghan TW, Kroenke K. Impact of pain on depression treatment response in primary care. Psychosom Med. 2004;66:17-22.

17. Garcia-Cebrian A, Bauer $\mathrm{M}$, Montejo $\mathrm{AL}$, et al. Factors influencing depression endpoints research (FINDER): study design and population characteristics. Eur Psychiatry. 2008;23:57-65.

18. Bauer M, Monz BU, Montejo AL, et al. Prescribing patterns of antidepressants in Europe: results from the Factors Influencing Depression Endpoints Research (FINDER) study. Eur Psychiatry. 2008;23:66-73.

19. Reed C, Monz BU, Perahia DGS, et al. Quality of life outcomes among patients with depression after 6 months of starting treatment: results from FINDER. J Affect Disord. 2009;113:296-302.

20. Black N. Why we need observational studies to evaluate the effectiveness of health care. BMJ. 1996;312:1215-8.

21. Zigmond AS, Snaith RP. The Hospital Anxiety and Depression Scale. Acta Psychiatr Scand. 1983;67: 361-70.

22. Olsson I, Mykletun A, Dahl AA. The hospital anxiety and depression rating scale: a cross-sectional study of psychometrics and case finding abilities in general practice. BMC Psychiatry. 2005;5:46.

23. Goldstein DJ, Lu Y, Detke MJ, Hudson J, Iyengar S, Demitrack MA. Effects of duloxetine on painful physical symptoms associated with depression. Psychosomatics. 2004;45:17-28.

24. Collins SL, Moore A, McQuay HJ. The visual analogue pain intensity scale: what is moderate pain in millimetres? Pain. 1997;72:95-7.
25. Ware JE Jr., Kosinski M, Keller SD. SF-36 Physical and mental health summary scales: a user's manual. Boston: Health Assessment Laboratory, New England Medical Center; 1994:8:21; 8:29.

26. Kind P, Hardman G, Macran S. UK Population norms for EQ-5D. York Centre for Health Economics Discussion Paper 172; 1999.

27. Daly EJ, Trivedi MH, Wisniewski SR, et al. Healthrelated quality of life in depression: a STAR ${ }^{\star} \mathrm{D}$ report. Ann Clin Psychiatry. 2010;22:43-55.

28. Munoz RA, McBride ME, Brnabic AJ, et al. Major depressive disorder in Latin America: the relationship between depression severity, painful somatic symptoms and quality of life. J Affect Disord. 2005;86:93-8.

29. Garcia-Campayo J, Ayuso-Mateos JL, Caballero L, et al. Relationship of somatic symptoms with depression severity, quality of life, and health resources utilization in patients with major depressive disorder seeking health care in Spain. Prim Care Companion J Clin Psychiatry. 2008;10:355-62.

30. Demyttenaere K, Reed C, Quail D, et al. Presence and predictors of pain in depression: results from the FINDER study. J Aff Disord. 2010 (Epub ahead of print).

31. Bair MJ, Robinson RJ, Katon W, Kroenke K. Depression and pain comorbidity: a literature review. Arch Intern Med. 2003;163:2433-45.

32. Sapin C, Fantino B, Nowicki ML, Kind P. Usefulness of EQ-5D in assessing health status in primary care patients with major depressive disorder. Health Qual Life Outcomes. 2004;2:20.

33. Sobocki P, Ekman M, Ågren H, et al. Health-related quality of life measured with EQ-5D in patients treated for depression in primary care. Value Health. 2007;10:153-60.

34. Kelly AM. The minimum clinically significant difference in visual analogue scale pain score does not differ with severity of pain. Emerg Med J. 2001;18:205-7.

35. National Institute for Health and Clinical Excellence (NICE). Depression: management of depression in primary and secondary care-NICE Guidance: CG23; 2004. http://guidance.nice.org. uk/CG23. Accessed 15 Feb 2013.

36. Leuchter AF, Husain MM, Cook IA, et al. Painful physical symptoms and treatment outcome in major depressive disorder: a STAR*D (Sequenced Treatment Alternatives to Relieve Depression) report. Psychol Med. 2010;40:239-51. 
37. The NHS information centre for health and social care. http://www.dh.gov.uk/en/Healthcare/Primary care/Primarycarecontracting/QOF/DH_4125653. Accessed 11 Feb 2013.

38. Kendrick T, Chatwin J, Dowrick C, et al. Randomised controlled trial to determine the clinical effectiveness and cost-effectiveness of selective serotonin reuptake inhibitors plus supportive care, versus supportive care alone, for mild to moderate depression with somatic symptoms in primary care: the THREAD (THREshold for AntiDepressant response) study. Health Technology Assessment. 2009;13:iii-iv, ix-xi, $1-159$. 